\title{
UTILIZAÇÃO DA ESPÉCIE Tradescantia pallida cv. purpurea COMO BIOINDICADORA DA QUALIDADE DO AR, ATRAVÉS DE BIOENSAIO DE MICRONÚCLEO
}

\author{
Luana Aparecida DUARTE XISTO ${ }^{1}$
}

Nicolle MARTINS TEBET MORGAN²

Nobel PENTEADO DE FREITAS ${ }^{3}$

\begin{abstract}
${ }^{1}$ Graduada em Engenharia Ambiental,Universidade de Sorocaba, Campus Cidade Universitária, Sorocaba, SP. luana_xisto@hotmail.com

${ }^{2}$ Graduada em Engenharia Ambiental, Universidade de Sorocaba, Campus Cidade Universitária, Sorocaba, SP. nii_tebet@hotmail.com

${ }^{3}$ Coordenador dos Cursos de Ciências Biológicas e Gestão Ambiental, Universidade de Sorocaba, Campus Cidade Universitária, Sorocaba, SP. nobel.freitas@ prof.uniso.br
\end{abstract}

Recebido em: 12/08/2016 - Aprovado em: 10/12/2017 - Disponibilizado em: 30/12/2017

\begin{abstract}
RESUMO
O desenvolvimento econômico e os crescentes impactos ambientais refletem significativamente na qualidade de vida do nosso meio, devido à produção e liberação de gases poluentes na atmosfera. Atualmente utiliza-se o biomonitoramento para identificar os efeitos da poluição por meio de organismos vivos, mediante a capacidade das espécies bioindicadoras em absorver e modificar substâncias tóxicas atmosféricas, pela sua maior sensibilidade à poluição que animais e homens. O objetivo deste trabalho foi o de utilizar a espécie Tradescantia pallida $\mathrm{cv}$. purpurea, no biomonitoramento das alterações da qualidade do ar através dos efeitos genotóxicos dos principais poluentes atmosféricos na cidade de Sorocaba/SP, por meio de bioensaio TRAD-MCN. Para a coleta de dados, foram selecionados três pontos amostrais, sendo um na periferia (ponto controle) e os demais na região central, próximos às estações de monitoramento do ar da CETESB. Durante o período de exposição, entre Agosto/2015 à Janeiro/2016, as frequências médias foram determinadas em tétrades jovens de células-mães de grãos de pólen, sendo expressas como MCN/100 células, obtendo como resultados significativamente superiores de genotoxicidade na região central do que no ponto controle, com mínima de $(1,33 \pm 0,11)$ no ponto controle e na região central máxima de $(4,33 \pm 0,11)$. A diferença entre as frequências médias obtidas com a análise microscópica comprova a capacidade da espécie Tradescantia pallida cv. purpurea ser considerada como bioindicadora da qualidade do ar. Desta forma, a utilização da espécie em bioensaios de micronúcleo, se mostra como uma possibilidade promissora para bioindicação da qualidade do ar em áreas urbanas.
\end{abstract}

Palavras-chave:Bioindicador; genotoxicidade; micronúcleo; poluição atmosférica; TRAD-MCN.

\section{UTILIZATION OF THE SPECIESTradescantia pallida cv. purpurea AS AIR QUALITY BIOINDICATOR, THROUGH BIOASSAY MICRONUCLEUS.}

\begin{abstract}
Economic development and growing environmental impacts reflect significantly the quality of life of our environment due to the production and release of greenhouse gases into the atmosphere. Currently biomonitoring is used to identify the effects of pollution by living organisms, by the ability of bioindicators to absorb and modify atmospheric toxic substances, due to their greater sensitivity to pollution that animals and men. The objective of this study was to use the Tradescantia pallida species $c v$. purpurea, the biomonitoring of changes in air quality through the genotoxic effects of major air pollutants in the city of Sorocaba / SP, through bioassay TRAD-MCN. To collect data, three sampling points were select; one in the periphery (control point) and the other in the central region, near the monitoring stations air CETESB. During the exhibition period from August / 2015 to January / 2016, the average frequencies were determined in tetrads young mother cells of pollen grains, and expressed as MCN / 100 cells, obtaining as significantly superior results of genotoxicity in central than in the control point with a minimum of $(1.33 \pm 0.11)$ in the control point and the maximum central region $(4.33 \pm 0.11)$. The difference between the mean frequency obtained from the microscopic analysis demonstrates the ability of Tradescantia pallida species $c v$. purpurea be considered as a bioindicator of air quality. Thus, the use of bioassays micronucleus species, shown as a promising possibility for air quality in urban areas bioindicators.
\end{abstract}

Keywords:Bioindicator; genotoxicity; micronucleus; atmospheric pollution; TRAD-MCN 


\section{INTRODUÇÃO}

Com o constante aumento populacional, industrial e o consumo desenfreado dos recursos não renováveis, as alterações ambientais refletem em crescentes impactos sociais, econômicos, tecnológicos e ambientais, resultando em danos ecológicos e, consequentemente, agravando a saúde e a qualidade de vida da população, levando a produção e liberação de diferentes gases na atmosfera alterando o equilíbrio entre seus componentes, dando se a isso o nome de poluição do ar.

Os centros urbanos são as regiões mais atingidas pelos impactos ambientais, pois abrigam grande quantidade de pessoas, há um intenso fluxo de automóveis, é onde se localizam várias indústrias e com isso diminuição das áreas verdes, consequentemente um aumento da poluição (LUIZ, 2004).

Bioindicadores são organismos que reagem às diversas variações do ambiente, alterando suas funções ou composições, sendo utilizadas para diversos monitoramentos (LIRA, ALMEIDA, PERES e SANTOS, 2008). A utilização de vegetais como organismos bioindicadores de poluentes atmosféricos, é adotada por certos estudos devido ao destaque do efeito genotóxico, onde é empregado a regularidade de micronúcleo em células-mãe de grãos de pólen da espécie Tradescantia pallida cv. purpurea como parâmetro considerado.
Assim, o presente trabalho fundamenta-se em verificações biológicas da qualidade do ar, por meio do bioensaio TRAD-MCN, utilizando como bioindicador a espécie vegetal Tradescantia pallida $c v$. purpurea. Procedimento esse que tem sido empregado em muitos trabalhos e pesquisas científicas a fim de conceituar a eficiência do potencial genotóxico dos poluentes atmosféricos.

Direcionado para a avaliação desse efeito genotóxico, as análises serão realizadas através das inflorescências da espécie Tradescantia pallida $c v$. purpurea como organismo experimental, em virtude de suas características genéticas.

\section{REVISÃO BIBLIOGRÁFICA}

A poluição do ar pode ser definida como a presença ou lançamento, no ambiente atmosférico, em concentrações de substâncias suficientemente altas que interfiram direta ou indiretamente na saúde, na segurança e no bem estar do homem, ou no uso de sua propriedade. As substâncias só são consideradas poluentes se provocarem efeitos mensuráveis tanto no homem, como nos animais e nas plantas, ou em materiais. Com isso, pode-se dizer que haverá poluição atmosférica sempre que houver qualquer variação na composição do ar com modificações físicas e químicas deste, de forma detectável pelos que vivem nele (MOUVIER, 1995). 
Nos últimos anos, o nível de compostos xenobióticos vem aumentando em todo o ecossistema, como resultado da atividade antropogênica sobre o meio ambiente, reduzindo a qualidade ambiental, comprometendo a saúde de todos os seres vivos (CAJARAVILLE et al, 2000).

Poluentes no ar prejudicam vegetais previsivelmente. Precisando respirar, os vegetais transformam a energia luminosa em energia química, adquirindo grande parte de constituintes químicos pelo sistema de raízes. A poluição atmosférica atinge a estrutura da folha destruindo células, bloqueando o transporte de energia dentro do vegetal, acrescentando novas composições ao solo. Cada planta tem individualidades quanto a sua reação, apresentando variações de quantidade de água disponível, condições do solo e sua fertilidade, temperatura e intensidade da luz (SEWELL, 1978).

Constantemente há a preocupação com as questões ambientais, dados estimam que existam 3,3 milhões de mortes causadas todo ano pela poluição atmosférica (ONU-BR, 2013).

Ainda segundo SEWELL, comumente árvores e outras plantas são utilizadas para melhoria da qualidade do ar. Por sua capacidade de "varrer" os poluentes do ar, as árvores impedem fisicamente o movimento ou quimicamente absorvendo-os.

O biomonitoramento é uma metodologia que consiste em identificar a poluição em organismos vivos, monitorando sua ação nos organismos bioindicadores, frequentemente utilizada na avaliação qualitativa e quantitativa da contaminação atmosférica, oferecendo assim diversas vantagens a população envolvida (LIMA, 2001).

Os bioindicadores vegetais podem ser utilizados de forma ativa ou passiva. $\mathrm{Na}$ forma ativa as espécies são previamente preparadas e posteriormente expostas no local de estudo e na forma passiva o monitoramento se faz com as espécies que já habitam o local (MAKI, SHITSUKA, BARROQUEIRO, 2013).

Segundo ALVES, a importância em avaliar a qualidade do ar atmosférico, através do biomonitoramento consiste no fato de que sem o ar, resistimos apenas alguns minutos, sendo assim, atualmente é recomendado realizar testes de natureza biológica, química e física, para verificação dos efeitos tóxicos em nosso meio.

Em locais onde as concentrações de poluentes são altas, podem ocasionar efeitos genotóxicos não apenas em humanos, mas também em animais e plantas, comprometendo o ecossistema (ISIDORI et al., 2003).

A bioindicação pode ser definida como a utilização de plantas para avaliação dos efeitos de diversas formas de poluição ambiental, sendo o seu uso bastante eficaz para avaliar efeitos da poluição do ar, 
fornecendo informações complementares aos danos causados aos seres vivos, revelando a presença ou ausência de poluentes (KLUMPP, 2001).

As plantas são mais sensíveis à poluição que os animais e os homens, sendo empregadas na avaliação de genotoxicidade de agentes químicos, indicando importantes fatores para controle da poluição do ar (ALVES et al., 2001).

Dentre os organismos bioindicadores de qualidade do ar, destaca-se a espécie Tradescantia pallida $c v$. purpurea, encontrada em diversos canteiros e jardins, é uma espécie tetraploide, resistente a parasitas e insetos, que floresce durante todo o ano, ela é originária do leste do Golfo do México e se caracteriza pela adaptabilidade a diversas condições climáticas (CARVALHO, 2005). Conhecida pelo nome popular de Coração Roxo, pelo fato das plantas serem de fácil manuseio, cultivo e cuidados, demonstrando respostas de fácil avaliação (LIRA, ALMEIDA, PERES e SANTOS, 2008).

A espécie Tradescantia pallida $c v$. purpurea, utilizada como bioindicadora da qualidade do ar, tem sido empregada em diversos trabalhos científicos, apresentando eficiência para avaliar o potencial genotóxico de poluentes aéreos, através do bioensaio TRAD-MCN (COPELLI, 2011). A Tradescantia pallida $c v$. purpurea, pertence à família Commelinaceae, ordem Commelinales e gênero Commelina, é considerada uma monocotiledônea de distribuição cosmopolita (JOLY, 1977).

Para análise da espécie bioindicadora, serão utilizados bioensaios desenvolvidos com inflorescências de Tradescantia pallida cv purpurea, sendo o teste de micronúcleo (TRAD-MCN) o mais utilizado (SAVOIA, 2007).

$\mathrm{O}$ teste de micronúcleo foi desenvolvido, no Brookhaver National Laboratory - EUA, sendo especificado por Te Hsiu Ma em 1976 (SISENANDO et al, 2009), é realizado a partir das células na fase tétrade, as quais são quatro células haploides, diferenciando posteriormente em grãos de pólen (RAVEN, EVERT, EICHHORN, 1996).

O bioensaio TRAD-MCN, se baseia na contagem de micronúcleos em células-mãe de grãos de pólen, onde determina a capacidade de substâncias causarem danos ao material genético, em consequência à formação de micronúcleos ao fim da divisão celular. A elevada formação de micronúcleos indica a presença de poluentes no local. O que causa danos ao material genético das plantas ocasionará danos aos humanos (LIMA, 2001).

\section{OBJETIVOS}

\section{OBJETIVO GERAL}

O presente trabalho tem como objetivo principal avaliar a eficiência do biomonitoramento das alterações da qualidade do ar através dos efeitos genotóxicos dos principais poluentes atmosféricos na cidade 
de Sorocaba -SP, por meio de análise de micronúcleo em inflorescências da espécie Trandescantia pallida cv purpurea.

\section{OBJETIVOS ESPECÍFICOS}

$\checkmark$ Comprovar a eficiência da espécie Trandescantia pallida $c v$. purpureacomo bioindicadora da qualidade do ar como potencial genotóxico através de bioensaio TRAD-MCN;

Avaliar a presença e a regularidade de micronúcleos nas inflorescências da espécie ao longo do período de exposição em pontos específicos de controle no município de Sorocaba - SP, como controle de incidência da poluição e alteração da qualidade do ar;

$\checkmark$ Desenvolver o biomonitoramento com a espécie vegetal como instrumentos para trabalhos científicos e de desenvolvimento;

$\checkmark$ Associar a relação da presença de micronúcleos das amostras aos índices da qualidade do ar vinculada à zona de exposição, junto aos parâmetros obtidos pela estação de monitoramento da qualidade do ar da CETESB - Companhia Ambiental do Estado de São Paulo, na cidade de Sorocaba.

\section{MATERIAIS E MÉTODOS}

Inicialmente as análises foram aprofundadas para definição do local de estudo e da espécie vegetal a ser utilizada como bioindicadora. Devido a espécie Tradescantia pallida $c v$. purpurea apresentar qualidades como fácil cultivo, florescência o ano todo e de fácil utilização pública, sendo então estabelecida como o objeto de estudo.

Após a definição da espécie a ser utilizada como bioindicadora, foi necessário realizar os seguintes procedimentos, montagem das floreiras, exposição nos locais de estudo, análise microscópica e avaliação dos resultados.

O município de Sorocaba possui uma área de $449 \mathrm{~km}^{2}$, localiza-se a $90 \mathrm{~km}$ a oeste da capital paulista, com uma população de aproximadamente $600 \mathrm{mil}$ habitantes, o seu relevo pode ser considerado levemente ondulado a ondulado, com altitude média de $600 \mathrm{~m}$, podendo chegar a $1.028 \mathrm{~m}$. Sua temperatura gira em torno de $12^{\circ} \mathrm{C}$ no inverno e de $30^{\circ} \mathrm{C}$ no verão, a precipitação média anual é de aproximadamente $1.350 \mathrm{~mm}$, a umidade relativa do ar, atinge $15 \%$ no período seco, principalmente no mês de setembro. (CETESB, 2015)

Para a determinação dos pontos de exposição das mudas e verificação de poluentes atmosféricos, foi levado em consideração o fato do município de Sorocaba conter duas estações de monitoramento da qualidade do ar controladas pela CETESB.

A estação de monitoramento da qualidade do ar manual de Sorocaba verifica apenas o parâmetro fumaça, enquanto a estação automática verifica os seguintes parâmetros: partículas inaláveis $\left(\mathrm{MP}_{10}\right)$, monóxido de nitrogênio (NO), dióxido de nitrogênio $\left(\mathrm{NO}_{2}\right)$, óxidos de nitrogênio $\left(\mathrm{NO}_{\mathrm{x}}\right)$, 
ozônio $\left(\mathrm{O}_{3}\right)$, umidade relativa do ar, temperatura, velocidade do vento e direção do vento (DV).

As floreiras foram expostas junto ou próximo às estações de monitoramento da qualidade do ar, considerando maior segurança e integridade do objeto de estudo, não ficando livre o acesso à população.

A estação automática se localiza na Escola Estadual Monsenhor João Soares, Rua Nhonhô Pires, $\mathrm{n}^{\mathrm{o}}$ 260, Vila Lucy, com Coordenadas UTM - 23K 2468417398675. Foi adotado o Condomínio Edifício San Raphael, na Rua José Gagliardi, nº 100, Vila Carvalho, onde as mudas ficaram expostas na sacada do $6^{\circ}$ andar, com Coordenadas UTM 23k 248989 7399551, próximo à estação manual localizada na Praça Dr. Artur Fajardo (Praça do Canhão).

As mudas foram produzidas no NEAS - Núcleo de Estudos Ambientais, pertencente à Universidade de Sorocaba, em abril a maio de 2015, sendo o local adotado como ponto controle para parâmetros de comparação. Foram preparados sacos plásticos ideais para plantio, com mudas da espécie Tradescantia pallida $c v$. purpurea, após o período de exposição, completo amadurecimento e formação, elas foram transplantadas em junho e julho de 2015 para as floreiras, e posteriormente levadas aos locais de exposição.

As floreiras foram distribuídas da seguinte forma, 5 (cinco) floreiras para o ponto de exposição do Condomínio Edifício San Raphael, 5 (cinco) floreiras para o ponto de exposição da estação de monitoramento da qualidade do ar automática de Sorocaba e 5 (cinco) floreiras para o ponto controle no NEAS.

Durante os meses de julho a agosto as floreiras foram expostas nos pontos definidos, sendo visitadas quinzenalmente para observação e coleta das inflorescências.

Posteriormente à fase de exposição e a coleta, foram comparadas as amostragens obtidas com a relação da qualidade do ar analisada pelas estações de monitoramento do órgão ambiental, podendo assim verificar a eficiência da espécie.

As mudas foram analisadas em bioensaio TRAD-MCN, prezando dois períodos climáticos distintos, onde encontrase maior variação da presença de poluentes atmosféricos, sendo o primeiro nos meses de agosto, setembro e outubro, onde o período de estiagem favorece a concentração de poluentes, e sequencialmente nos meses de novembro, dezembro e janeiro, quando as chuvas alteram as características ambientais.

As inflorescências são preparadas e analisadas em microscopia óptica, verificando a presença de micronúcleos.

Após a coleta, os botões são inseridos em solução de Carnoy (solução aceto-etanol 1:3) por um período de $24 \mathrm{~h}$, e posteriormente é feita uma imersão em etanol $70 \%$ e estocadas a $4^{\circ} \mathrm{C}$ até o momento da análise. 
Para a análise microscópica, as inflorescências são dissecadas, e posteriormente são maceradas com auxílio de estilete histológico sobre lâminas de vidro, onde são descartados os vestígios das anteras, então são corados com corante carmim acético. Em seguida, a lamínula é sobreposta e são passadas as lâminas sobre a chama do bico de Bunsen para estabilização do corante, as lâminas prontas são avaliadas em microscopia óptica sob aumento de 400x.

Para detecção da alteração mutagênica, são preparadas 5 (cinco) lâminas por mês em cada ponto de exposição, totalizando 15 (quinze) lâminas mensais, sendo necessário que para cada lâmina seja analisada 300 tétrades. Durante a contagem das tétrades, é feita a contagem dos micronúcleos presentes nas mesmas células. O número de micronúcleos presentes por lâmina verifica a presença de poluentes no ar, onde a planta foi exposta.

\section{RESULTADOS E DISCUSSÃO}

Segundo dados disponibilizados no site da CETESB, foi possível observar que há uma variação muito grande em relação aos índices de qualidade do ar, durante os meses de julho de 2015 a janeiro de 2016. E que grande parte está relacionada com a temperatura e umidade relativa diária, quanto maior a temperatura e menor a umidade relativa do ar, maior a concentração de poluentes na atmosfera.
As médias mensais dos valores obtidos no site da CETESB, estão dentro do parâmetro de qualidade do ar, não ultrapassando o índice de $40 \mu \mathrm{g} / \mathrm{m}^{3}$, sendo considerado como boa, porém alguns dias esses valores ultrapassaram a média sendo também considerada como qualidade moderada, ruim e muito ruim.

Vale ressaltar que estes valores estando dentro do parâmetro de qualidade não significam que o índice de qualidade do ar seja realmente boa, e sim que os valores são considerados como aceitáveis as nossas condições, não ocasionando prejuízos à saúde.

Durante o período de exposição, as frequências médias foram determinadas em tétrades jovens de células-mães de grãos de pólen, sendo expressas como MCN/100 células, obtendo como resultados significativamente superiores de genotoxicidade na região central do que no ponto controle, destacando o mês de agosto com mínima de $(2,47 \pm 0,33)$ no ponto controle e na região central máxima de $(4,33$ $\pm 0,11$, como mostra a tabela 1 e o gráfico 1 .

Tabela 1 - Frequência média mensal de micronúcleos (MCN/100 células \pm desvio padrão), durante os meses de exposição no município de Sorocaba/SP.

\begin{tabular}{|c|c|c|c|}
\hline \multirow{2}{*}{ Meses } & \multicolumn{3}{|c|}{ Pontos Amostrais } \\
\cline { 2 - 4 } & Escola & Condomínio & NEAS \\
\hline Ago/15 & $4,33 \pm 0,11$ & $4,20 \pm 0,19$ & $2,47 \pm 0,33$ \\
\hline Set/15 & $3,53 \pm 0,22$ & $3,13 \pm 0,18$ & $2,20 \pm 0,15$ \\
\hline Out/15 & $3,07 \pm 0,19$ & $3,73 \pm 0,17$ & $1,93 \pm 0,13$ \\
\hline Nov/15 & $3,27 \pm 0,16$ & $3,07 \pm 0,09$ & $1,87 \pm 0,19$ \\
\hline Dez/15 & $2,67 \pm 0,15$ & $3,00 \pm 0,35$ & $1,33 \pm 0,11$ \\
\hline Jan/16 & $2,93 \pm 0,21$ & $2,93 \pm 0,22$ & $1,67 \pm 0,13$ \\
\hline
\end{tabular}

Fonte: Autores. 
Gráfico 1 - Frequência média mensal de micronúcleos (MCN/100 células), durante os meses de exposição no município de Sorocaba/SP.

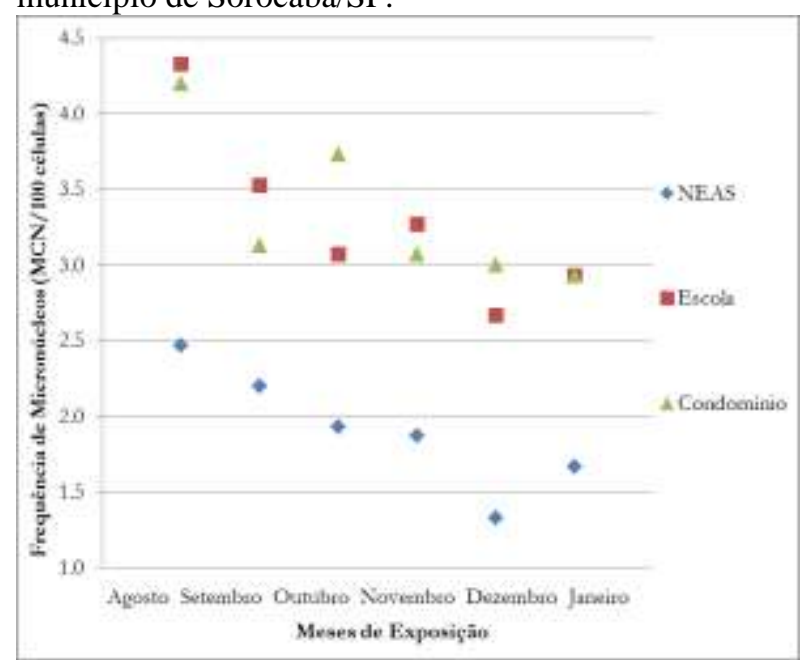

Fonte: Autores.

Ocorreu um número significativo de micronúcleos nos pontos de exposição, sendo observado no período de estiagem frequências mais elevadas de micronúcleos nas célulasmãe de grãos de pólen.

Com relação aos dados obtidos diariamente no site da CETESB, é possível comparar que os períodos com maior concentração de poluentes na atmosfera é o mesmo período com maior número de micronúcleos presentes nas células.

O bioensaio TRAD-MCN é eficiente para avaliar danos ao material genético por diferentes poluentes atmosféricos, sendo avaliados através da frequência de micronúcleos. (MA, 1984).

Segundo BLUME et al, a qualidade do ar de Sapucaia do Sul, apresentou alto potencial genotóxico durante o período de exposição, comprovando que as frequências mais elevadas de micronúcleos, foram significativamente superiores do que as frequências nos pontos controles.

SAVÓIA estabelece que precipitação, umidade relativa do ar e temperatura são fatores que interferem nas frequências de micronúcleos durante o período de exposição. Estudos na cidade de Taubaté apontam que a média de micronúcleos encontradas em espécies expostas a poluentes do ar,na região central foi significativamente superior que na região periférica.

Portanto as frequências médias obtidas com a análise microscópica comprovam a capacidade da espécie Tradescantia pallida cv. purpurea ser considerada como bioindicadora da qualidade do ar.

\section{CONCLUSÃO}

Os resultados obtidos neste estudo sobre o uso da espécie Tradescantia pallida $c v$. purpurea como bioindicadora vegetal no monitoramento da poluição atmosférica se revelaram eficiente na avaliação da genotoxicidade.

Os valores atingidos durante o período de exposição se provaram confiáveis e coerentes. Somente no mês de outubro de 2015 a variância obtida foi significativa ao nível de 5\% de probabilidade, nos outros meses ela não se apresentou a mesma relevância, entretanto ficou nítido a diferença entre a região central e a periférica, enfatizando a sensibilidade da espécie através do método TRAD-MCN utilizado. 
A vantagem da aplicação da espécie em estudo se mostrou de grande potencial e efetividade devido ao fato de ser de sistema rápido, baixo custo, baixa complexidade de interpretação e execução dos bioensaios, se revelando uma ferramenta de biomonitoramento a ser adotada como conhecimento científico.

\section{REFERÊNCIAS}

ALVES, E.S. et al. Biomonitoramento indoor do potencial mutagênicos do ar em laboratórios e herbário do Instituto de Botânica por meio do bioensaio TRAD-MCN. Hoehnea, Sao Paulo, v. 30, n. 2, p. 89-94, 2001.

ALVES, N.O. Genotoxicidade e composição do material particulado emitido pela queima de biomassa: um estudo de caso me Tangará da Serra, região da Amazônia brasileira. 2010. 82f. Dissertação (Mestrado em Bioquimíca) Universidade Federal do Rio Grande do Norte, Natal, 2010.

BLUME, K.K., COSTA, G.M., CASSANEGO, M.B.B., DROSTE, A. Genotoxicidade do ar em área urbana na região metropolitana de Porto Alegre, RS, Brasil. Revista Brasileira de Biociências, p. 158-163, 2014.

CAJARAVILLE, M.P., BEBIANNO, J.M., BLASCO, J., PORTE C., SARASQUETE. C., VIARENGO A. The use of biomarkers to assess the impact of pollution in coastal environments of the Iberian Peninsula: a practical approach. Sci Total Environ 2000; 247:295-311

CARVALHO, H.A. A Tradescantia como bioindicador vegetal na monitoração dos efeitos clastogênicos das radiações ionizantes. RadiolBras v.38 n.6, São Paulo, 2005, P.459462.Disponível em:http:// www.scielo.br/cgi- bin/wxis.exe/iah/. Acesso em: 03 de Abril de 2007.

CETESB. Relatório da qualidade do ar no Estado de São Paulo. Disponível em: http://www.cetesb.sp.gov.br/userfiles/file/ar/r elatorios/relatorio-ar-2014.pdf. Acesso em: 03 de junho de 2015.

COPELLI, T.S. Biomonitoramento da qualidade do ar utilizando ensaio de micronúcleo em Tradescantia sp. Curitiba. 2011

ISIDORI, M., FERRARA, M., LAVORGNA, M., NARDELLI, A., PARRELLA, A. (2003) In situ monitoring of urban air in Southern Italy with the Tradescantia micronucleus bioassay and semipermeable membrane devices (SPMDs). Chemosphere 52: 121-126.

JOLY, AYLTON, B. Botânica: Introdução a taxonomia vegetal. $4^{\mathrm{a}}$ edição, São Paulo, 1977.

KLUMPP, A. Utilização de bioindicadores de poluição em condições temperadas e tropicais. In: N. B. M. MAIA, H. L.; BARELLA, W. (Ed.). Indicadores ambientais: conceitos e aplicações. São Paulo: Educ/Comped/Inep, p.77 - 94. Utilização de bioindicadores de poluição atmosférica em condições temperadas e tropicais. 2001.

LIMA, J. S. Processos biológicos e o biomonitoramento: aspectos bioquímicos e morfológicos. In: Maia, N. B. Martos, H. L. \&Barrella, W. Indicadores ambientais: conceitos e aplicações. São Paulo: EDUC, 2001. p.95-115.

LIRA, O.F.C.; ALMEIDA, N.N.; PERES, W.L.; SANTOS, W.S. Projeto Piloto de Biomonitoramento com Tradescantia pallida em munícipios com Alto Risco Ambiental. Cuiabá, 2008.

LUIZ, E.J.; LAVENDOWSKI, I.M.F.; OLIVEIRA, G. P.; GUIMARÃES, E.T.; DOMINGOS, M.; SALDIVA, P.H.N. Sentindo a Cidade: Biomonitoramento da 
Qualidade do Ar de Santo André com plantas da espécie Tradescantia pallida e a Educação Ambiental. Santo André, 2004.

MAKI, E.S., SHITSUKA, R., BARROQUEIRO, C.H., SHITSUKA, D.M.

Utilização de Bioindicadores em monitoramento de Poluição, 2013.

MA, T.H. Tradescantia-Micronucleus (TRAD-MCN) tests on 140 health-related agents. Mut. Res., Amsterdam, v. 138, n. 2, p. 157-167, 1984.

ONU-BR. Consequências da poluição do ar são piores do que estimativas anteriores, alerta OMS. Website das Nações Unidas no Brasil. Publicado em: 09 de abril de 2013 e disponível em: http://www.onu.org.br/consequencias-dapoluicao-do-ar-sao-piores-do-que-estimativasanteriores-alerta-oms/. Acesso em: 03 de junho de 2015

RAVEN, PETER H.; EVERT RAY, F. EICHHORN, SUSAN, E. Biologia Vegetal. $5^{\text {a }}$ edição, Guanabara, Rio de Janeiro, 1996.

SAVOIA, Eriane, L. J. Potencial de Tradescantiapallida cv. purpurea para biomonitoramento da poluição aérea de Santo André - São Paulo, por meio do bioensaioTrad - MCN e do acúmulo foliar de elementos tóxicos. 2007 118f. Dissertação (Mestrado em Ciências). Faculdade de Medicina da Universidade de São Paulo Área de concentração de Fisiopatologia experimental, São Paulo, 2007.

SEWELL, GRANVILLE HARDWICK. Administração e controle da qualidade ambiental. São Paulo: EPU, 1978.

SISENANDO, H.A., BATISTUZZO DE MEDEIROS, S. R., HACON, S.S., Tradescantia pallida: Mais do que uma linda flor, um importante bioindicador da qualidade ambiental. Genética na escola, V. 04,02; 2009. 